OPEN ACCESS

Edited by:

Pramod Kumar Pal,

National Institute of Mental Health and Neurosciences (NIMHANS), India

Reviewed by: Renato Puppi Munhoz, Pontifical Catholic University of

Parana, Brazil Carlos Henrique Ferreira Camargo, Federal University of Paraná, Brazil

${ }^{*}$ Correspondence: Mazen Elkurd mazen.elkurd@utsouthwestern.edu

Specialty section: This article was submitted to Movement Disorders, a section of the journal Frontiers in Neurology

Received: 17 May 2021 Accepted: 02 July 2021 Published: 27 July 2021

Citation: Elkurd M, Wang J and Dewey RB Jr (2021) Lateralization of Motor Signs Affects Symptom Progression in Parkinson Disease.

Front. Neurol. 12:711045. doi: 10.3389/fneur.2021.711045

\section{Lateralization of Motor Signs Affects Symptom Progression in Parkinson Disease}

\author{
Mazen Elkurd ${ }^{1 *}$, Jijia Wang ${ }^{2}$ and Richard B. Dewey Jr. ${ }^{1}$ \\ ${ }^{1}$ Department of Neurology, UT Southwestern Medical Center, Dallas, TX, United States, ${ }^{2}$ Department of Applied Clinical \\ Research, UT Southwestern Medical Center, Dallas, TX, United States
}

Background: Asymmetry of motor signs is a cardinal feature of Parkinson disease which may impact phenotypic expression.

Objective: To investigate the relationship between lateralization of motor signs and symptom progression and severity during longitudinal observation for up to 4 years in a naturalistic study.

Methods: We analyzed data prospectively collected during the NINDS Parkinson Disease Biomarker Project (PDBP). We defined the Movement Disorder Society Revision of the Unified Parkinson Disease Rating Scale (MDS-UPDRS) part II as the primary measure of symptom progression. Left side predominant subjects were those whose lateralized motor scores on the MDS-UPDRS part III were $\geq 2$ points higher on the left side than on the right side of the body. Multiple regression models (controlled for age, gender, education years, ethnicity, levodopa equivalent daily dose (LEDD) at baseline, and years with $\mathrm{PD}$ ) were used to estimate the rate of symptom progression comparing left predominant (LPD) with non-left predominant (NLPD) subjects. A sensitivity analysis was performed using the same multiple regression models in the subgroups of low (0-26) or high (>27) MDS-UPDRS II score at baseline to determine if PD severity influenced the results.

Results: We included 390 participants, 177 LPD and 213 NLPD. We found that MDS-UPDRS part II progression from baseline to 48 months was faster in LPD compared to NLPD (0.6 points per year faster in LPD, $p=0.05$ ). Additionally, the LPD group was statistically significantly worse at baseline and at 48 months in several subparts of the MDS-UPDRS and the Parkinson's Disease Questionnaire-39 (PDQ-39) mobility score. Significantly slower progression (difference of $-0.8, p=0.01$ ) and lower score at 48 months (difference of $-3.8, p=0.003$ ) was seen for NLPD vs. LPD in the group with lower baseline MDS-UPDRS part II score.

Conclusion: Left side lateralization was associated with faster symptom progression and worse outcomes in multiple clinical domains in our cohort. Clinicians should consider using motor predominance in their counseling regarding prognosis.

Keywords: Parkinson's disease, lateralization, asymmetry, progression, motor symptoms 


\section{INTRODUCTION}

Parkinson disease is a progressive neurodegenerative condition of unknown etiology with no known cure. PD is a complex, heterogeneous condition which presents with various blends of motor and non-motor symptoms. These differing subtypes may vary considerably in rate of progression (1). This heterogeneity, combined with lack of any predictive tools, is an obstacle to accurate prognostic counseling of patients and complicates disease modifying research because different subtypes may respond differently to interventions. Identification of distinct phenotypic subtypes has proven an important area of research in the past decade (2), and numerous studies have been published to date examining the association of various motor and nonmotor features with disease progression (3). Despite these efforts, significant uncertainty and controversy remains, and no tool exists for predicting the trajectory of any individual case $(3,4)$.

Asymmetry of motor signs is an important feature of PD which has been investigated as a potential predictor of disease severity or progression. Asymmetry is a cardinal feature of the human brain, with well-understood lateralization of functions including specialized areas for language and visuospatial processing (5). Asymmetry has also been described in pathological states including several neurodegenerative processes such as frontotemporal dementia (6), Alzheimer disease (7), and Huntington disease (8). In Parkinson disease, this asymmetry is important for diagnostic certainty and when absent poses a red flag against the diagnosis (9). Motor asymmetry in PD has been substantiated by imaging (10) and histopathologic examinations (11) demonstrating asymmetric nigrostriatal degeneration correlating with lateralized severity.

The clinical significance of asymmetry of motor symptoms in PD has not been extensively studied though there are reports linking lateralized symptoms to severity of motor and non-motor symptomatology. Amick et al. found that individuals with left-predominant symptoms exhibited poorer visual memory, whereas those with right-predominant features demonstrated poorer verbal memory (12). Dewey et al. found that right-sided onset of tremor appeared to be predictive of a lower risk of depressive symptoms (13). Cubo et al. evaluated 652 PD patients and noted that left-predominant disease was associated with worse motor and non-motor performance (14). A major limitation of this prior work was its cross-sectional nature. These provide evidence that motor asymmetry is associated with function at a given point in time. By contrast, we undertook to use the prospectivelycollected dataset of the Parkinson Disease Biomarker Program (PDBP) to determine if motor laterality at baseline influenced future severity and progression during up to 4 years of follow-up. Based on prior work, our hypotheses were that (1) left-side predominant disease would be associated with worse motor and cognitive symptoms, and (2) that rate of progression of symptoms would be faster in those with left-side motor predominance.

\section{METHODS}

\section{Standard Protocol Approvals, Registrations, and Patient Consents}

For this study, we analyzed data previously collected through the National Institute of Neurologic Disorders and Stroke (NINDS) PDBP. This was a prospective, multi-site longitudinal cohort study in which clinical data and biological samples were collected from participants with Parkinson disease as well as control subjects who were followed for up to 4 years. The methods of data collection have been previously published (15). The study protocol was reviewed and approved by the Institutional Review Board of the University of Texas Southwestern Medical Center, and at the other institutions where subjects were recruited and followed as part of this project. Written informed consent was obtained from all participants. The study was registered on clinicaltrials.gov with registration number NCT01767818. The study is reported in accordance with STROBE reporting criteria for cohort studies.

\section{Subjects}

Subjects were recruited in this longitudinal cohort study at three sites in the United States: UT Southwestern Medical Center, Johns Hopkins University School of Medicine, and PennState Health Milton S. Hershey Medical Center between 2012 and 2017. Subjects had a diagnosis of idiopathic PD according to UK Brain Bank Criteria, were males or females aged 30 or older at the time of diagnosis, if untreated with dopaminergic agents had confirmation of dopamine transporter deficit by I-123 Ioflupane SPECT (DatScan), and if treated with dopaminergic agents had clinical evidence of a favorable response to treatment. Subjects suspected to have atypical parkinsonian disorders or secondary parkinsonian syndromes due to drugs, metabolic disorders or encephalitis were excluded from this analysis. Most subjects were initiated on anti-parkinsonian dopaminergic therapy at their physician's discretion prior to study entry, while a few entered the study unmedicated and were later placed on dopaminergic therapy. For this project, we included all available longitudinal data from baseline through 48 months, but due to a data anomaly in motor scoring introduced by a change of the clinical rater at one recruitment site after the month 12 assessment, we censored MDS-UPDRS part III data from 18 months onward from that site.

\section{Outcome Measures}

We designated the primary outcome measure for this analysis to be the rate of change per year in the MDS-UPDRS part II score from baseline to month 48. Though the subparts and total score of the MDS-UPDRS have been found to increase in a linear fashion in longitudinal cohorts and serve as reliable measures of disease progression $(16,17)$, we chose the part II score as the primary outcome measure because it has been demonstrated to have a more robust association with PD disease duration independent of medication status compared to other UPDRS subscores, and thus may be a stronger predictor of progression in Parkinson disease (18). In addition, we were 
able to avoid censoring this variable at later visits at the site where the change of rater occurred because this is a patientreported score. Secondary outcome variables were the rate of change from baseline to 48 months and average scores at 48 months of MDS-UPDRS I, III, IV and total scores (19), Montreal Cognitive Assessment (MoCA) (20), levodopa equivalent daily dose (LEDD) (21), Schwab \& England Activities of Daily Living Scale (S\&E) (22), a Global Composite Outcome (GCO) which combines part I-III of the MDS-UPDRS, S\&E and MoCA (23), Epworth Sleepiness Scale (ESS) (24), University of Pennsylvania Smell Identification Test (UPSIT) (25), Hamilton Anxiety Scale (HAM-A) (26), Hamilton Depression Rating Scale (HAM-D) (27), and the Parkinson's Disease Questionnaire-39 (PDQ-39) scores for mobility, activities of daily living (ADL), emotional well-being, stigma, social support, cognition, communication, and bodily discomfort (28).

\section{Determination of Motor Lateralization}

We used the MDS-UPDRS part III score at the baseline visit to determine lateralization. For each subject, we summed items 20-26 yielding a total lateralized motor score for each side of the body. Using a cut-off of $\geq 2$ points according to the method described by Poletti et al. (29) participants were assigned to either the left predominant (LPD) or nonleft predominant group (NLPD). The non-left predominant group consisted of those with right-predominant and symmetric motor scores.

\section{Statistical Analysis}

Two-sample $t$ test, Mann-Whitney $U$ test, and chi-square tests were used to compare clinical features at baseline. We used univariate linear regression to estimate the subject-specific rate of change per year in each outcome measure and from this, predicted measurements at 48 months. In the same manner, we estimated the subject-specific difference between LPD and NLPD of MDS-UPDRS II at each visit. We then employed multiple regression models to identify whether there was a difference between left and non-left predominant subjects while controlling for demographic (age, gender, education, and ethnicity) and clinical (LEDD at baseline and PD duration) variables. A sensitivity analysis was performed using the same multiple regression models in the subgroups of low (0-26) or high (>27) MDS-UPDRS II score at baseline to determine if PD severity influenced the results. All statistical analyses were carried out using SAS 9.4 (SAS Institute Inc, Cary, NC). The graph was generated using Prism version 8 (GraphPad Software, LLC).

\section{RESULTS}

\section{Baseline Demographic and Clinical Characteristics}

Our analysis included a total of 390 participants, of whom 177 were classified as left-predominant (LPD) and 213 were nonleft predominant (NLPD). The baseline demographic and clinical characteristics of the participants are shown in Table 1. Age, years of education, gender, and ethnicity were similar in both
TABLE 1 | Baseline characteristics of PD subjects by groups (mean \pm interquartile range).

\begin{tabular}{|c|c|c|c|}
\hline Variable & LPD & NLPD & $P$ value \\
\hline Age & $65 \pm 14$ & $64 \pm 13$ & 0.11 \\
\hline Education years & $16 \pm 5.0$ & $16 \pm 4.0$ & 0.53 \\
\hline Gender (M) & $62 \%$ & $56 \%$ & 0.25 \\
\hline Ethnicity (Hispanic or Latino) & $6 \%$ & $4 \%$ & 0.39 \\
\hline Years with PD* & $5.9 \pm 6.0$ & $5.0 \pm 6.0$ & 0.02 \\
\hline MDS-UPDRS part I & $9.0 \pm 8.0$ & $8.1 \pm 7.0$ & 0.09 \\
\hline MDS-UPDRS part $\|^{\star}$ & $10 \pm 10$ & $8.5 \pm 9.0$ & 0.02 \\
\hline MDS-UPDRS part III* & $25 \pm 17$ & $19 \pm 13$ & $<0.001$ \\
\hline MDS-UPDRS part IV* & $2.8 \pm 5.0$ & $2.0 \pm 3.0$ & 0.04 \\
\hline MDS-UPDRS total* & $47 \pm 31$ & $38 \pm 28$ & $<0.001$ \\
\hline MoCA & $26 \pm 4.0$ & $26 \pm 3.0$ & 0.39 \\
\hline$S \& E^{\star}$ & $0.85 \pm 0.10$ & $0.90 \pm 0.10$ & 0.02 \\
\hline PDQ-39 Mobility* & $17 \pm 23$ & $12 \pm 15$ & 0.05 \\
\hline PDQ-39 Activities of Daily Living & $17 \pm 21$ & $16 \pm 21$ & 0.61 \\
\hline PDQ-39 Emotional Well-being & $15 \pm 21$ & $13 \pm 21$ & 0.49 \\
\hline PDQ-39 Stigma & $15 \pm 25$ & $13 \pm 19$ & 0.54 \\
\hline PDQ-39 Social Support & $7.3 \pm 8.3$ & $5.6 \pm 8.3$ & 0.84 \\
\hline PDQ-39 Cognitive Impairment & $19 \pm 19$ & $17 \pm 19$ & 0.22 \\
\hline PDQ-39 Communication & $14 \pm 25$ & $13 \pm 17$ & 0.67 \\
\hline PDQ-39 Bodily Discomfort & $24 \pm 25$ & $23 \pm 25$ & 0.81 \\
\hline $\mathrm{GCO}^{*}$ & $0.12 \pm 0.80$ & $-0.12 \pm 0.68$ & 0.002 \\
\hline HAM-A & $6.5 \pm 6.0$ & $6.4 \pm 7.0$ & 0.69 \\
\hline HAM-D & $5.1 \pm 5.0$ & $4.6 \pm 4.0$ & 0.22 \\
\hline Epworth* & $8.1 \pm 6.0$ & $7.0 \pm 6.0$ & 0.03 \\
\hline UPSIT & $19.5 \pm 11.0$ & $20.0 \pm 12.0$ & 0.48 \\
\hline
\end{tabular}

Years with $P D$ is calculated from the time of diagnosis.

${ }^{*}$ Designates $p \leq 0.05$.

MoCA, Montreal Cognitive Assessment; S\&E, Schwab and England Activities of Daily Living Scale; GCO, global composite outcome; HAM-A, Hamilton Anxiety Scale; HAMD, Hamilton Depression scale; Epworth, Epworth Sleepiness Scale; UPSIT, University of Pennsylvania Smell Identification Test.

groups. PD duration (calculated from the time of PD diagnosis) was longer in the LPD group (5.93 vs. 4.98 years, $p=0.015$ ). MDS-UPDRS II, III, IV, and total scores were higher at baseline in the LPD group compared to NLPD. Participants in the LPD group also had significantly worse scores at baseline on the S\&E (0.85 vs. $0.90, p=0.016)$, ESS (8.14 vs. $7.04, p=0.03)$, PDQ39 mobility ( 16.7 vs. $11.5, p=0.05)$, and GCO (0.12 vs. -0.12 , $p=0.002)$.

\section{Rate of Progression}

The primary outcome (rate of change in points per year of the MDS-UPDRS part II score) just met statistical significance showing that the LPD group progressed 0.6 points faster per year than the NLPD group $(p=0.05)$. A graph of the mean MDSUPDRS part II score over time in the two groups is shown in Figure 1. We also performed exploratory analyses of all other outcome variables assessed and found no significant differences in the rate of progression between the groups in these other measures (Table 2). 


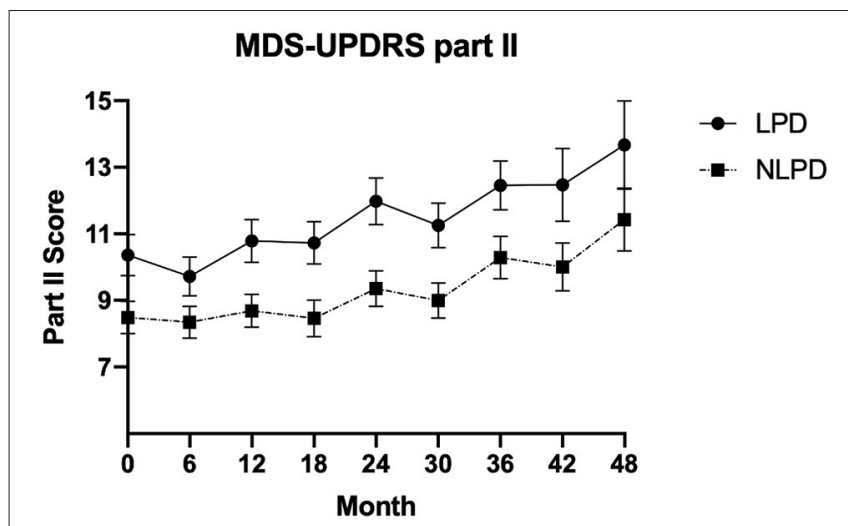

FIGURE 1 | Mean value of MDS-UPDRS part II score in the two groups over time. Error bars represent standard error of the mean.

TABLE 2 | Multiple linear regression analysis showing the difference in progression rate (defined by rate of change per year) as measured by various clinical endpoints between NLPD and LPD subjects.

\begin{tabular}{|c|c|c|}
\hline Outcome & Estimate of NLPD-LPD & $P$ value \\
\hline MDS-UPDRS part I & 0.22 & 0.37 \\
\hline MDS-UPDRS part II* & -0.62 & 0.05 \\
\hline MDS-UPDRS part III & 0.56 & 0.50 \\
\hline MDS-UPDRS part IV & -0.28 & 0.11 \\
\hline MDS-UPDRS total & -0.55 & 0.63 \\
\hline MoCA & 0.13 & 0.35 \\
\hline LEDD & 0.45 & 0.97 \\
\hline S\&E & 0.00 & 0.77 \\
\hline GCO & -0.03 & 0.36 \\
\hline Epworth & 0.31 & 0.10 \\
\hline UPSIT & 0.02 & 0.94 \\
\hline HAM-A & -0.26 & 0.34 \\
\hline HAM-D & 0.04 & 0.83 \\
\hline PDQ-39 Mobility & -0.93 & 0.26 \\
\hline PDQ-39 Activities of Daily Living & -0.37 & 0.61 \\
\hline PDQ-39 Emotional Well-being & -0.17 & 0.83 \\
\hline PDQ-39 Stigma & -0.24 & 0.78 \\
\hline PDQ-39 Social Support & -0.26 & 0.70 \\
\hline PDQ-39 Cognitive Impairment & 0.59 & 0.46 \\
\hline PDQ-39 Communication & -0.26 & 0.75 \\
\hline PDQ-39 Bodily Discomfort & -0.36 & 0.72 \\
\hline
\end{tabular}

Each model is adjusted for age, gender, education years, ethnicity, LEDD at baseline, and years with $P D$ (as measured from time of diagnosis).

*Designates $p \leq 0.05$.

MoCA, Montreal Cognitive Assessment; S\&E, Schwab and England Activities of Daily Living Scale; GCO, global composite outcome; HAM-A, Hamilton Anxiety Scale; HAMD, Hamilton Depression scale; Epworth, Epworth Sleepiness Scale; UPSIT, University of Pennsylvania Smell Identification Test.

\section{Symptom Severity at $\mathbf{4 8}$ Months}

At 48 months, the average MDS-UPDRS II score in the LPD group was 3.4 points higher than the NLPD group $(p=0.01)$. By contrast, the MDS-UPDRS parts I, III and total scores were not significantly different. The MDS-UPDRS part IV was 1.4 points
TABLE 3 | Multiple linear regression analysis showing difference in average measurement at 48 months between NLPD and LPD groups.

\begin{tabular}{lcc}
\hline Outcome & Estimate of NLPD-LPD & $\boldsymbol{P}$ value \\
\hline MDS-UPDRS part I & 0.18 & 0.86 \\
MDS-UPDRS part II & -3.4 & 0.01 \\
MDS-UPDRS part III & -2.7 & 0.41 \\
MDS-UPDRS part IV* & -1.4 & 0.03 \\
MDS-UPDRS total & -8.8 & 0.06 \\
MoCA & 0.45 & 0.44 \\
LEDD & -15 & 0.78 \\
S\&E & 0.03 & 0.16 \\
GCO & -0.25 & 0.07 \\
Epworth & 0.68 & 0.36 \\
UPSIT & -0.09 & 0.93 \\
HAM-A & -1.4 & 0.20 \\
HAM-D & -0.28 & 0.71 \\
PDQ-39 Mobility & -7.6 & 0.03 \\
PDQ-39 Activities of Daily Living & -2.4 & 0.41 \\
PDQ-39 Emotional Well-being & -2.1 & 0.46 \\
PDQ-39 Stigma & -2.7 & 0.38 \\
PDQ-39 Social Support & -3.1 & 0.19 \\
PDQ-39 Cognitive Impairment & 0.70 & 0.81 \\
PDQ-39 Communication & -2.3 & 0.46 \\
PDQ-39 Bodily Discomfort & -2.6 & 0.47 \\
\hline ECh & & \\
\hline
\end{tabular}

Each model is adjusted for age, gender, education years, ethnicity, LEDD at baseline, and years with $P D$ (as measured from time of diagnosis).

${ }^{\star}$ Designates $p \leq 0.05$.

MoCA, Montreal Cognitive Assessment; S\&E, Schwab and England Activities of Daily Living Scale; GCO, global composite outcome; HAM-A, Hamilton Anxiety Scale; HAMD, Hamilton Depression scale; Epworth, Epworth Sleepiness Scale; UPSIT, University of Pennsylvania Smell Identification Test.

higher $(p=0.03)$ in the LPD group, and the PDQ-39 mobility score was 7.6 points higher in the LPD group $(p=0.03)$. The remaining measures did not show any significant differences in average scores at 48 months (Table 3 ).

\section{Sensitivity Analysis}

We found significantly slower progression in the NLPD compared to LPD (difference of $-0.8, p=0.01$ ) for the low MDSUPDRS part II at baseline group, whereas there was no difference between NLPD and LPD in the high group $(-2.7, p=0.6)$. The average MDS-UPDRS II score at month 48 was significantly lower in NLPD in the low group $(-3.8, p=0.003)$ whereas no difference was seen between NLPD and LPD in the high group $(-8.6, p=0.6)$ (Table 4).

\section{DISCUSSION}

In this analysis of a large multi-center longitudinal cohort of PD subjects, we demonstrated that individuals with left-predominant PD motor symptoms exhibit more rapid symptom progression over a 48 month period as measured by MDS-UPDRS part II scores. Our data also confirmed previously reported observations that those with left-predominant disease have a higher disease 
TABLE 4 | Sensitivity analysis comparing rate of progression measured by change in MDS-UPDRS II score and average score at 48 months in LPD vs NLPD groups, stratified by baseline MDS-UPDRS II (high or low groups).

\begin{tabular}{lcc}
\hline & $\begin{array}{c}\text { LPD vs. NLPD } \\
\text { progression }\end{array}$ & $\begin{array}{c}\text { LPD vs. NLPD score at 48 } \\
\text { months }\end{array}$ \\
\hline Low baseline score & $-0.7587\left(p=0.013^{\star}\right)$ & $-3.7847\left(p=0.003^{\star}\right)$ \\
High baseline score & $-2.6966(p=0.57)$ & $-8.5878(p=0.599)$ \\
\hline${ }^{*}$ Designates $p \leq 0.05$. & &
\end{tabular}

*Designates $p \leq 0.05$.

burden when compared to those with opposite laterality or symmetric motor signs. Our finding that both symptom scores and PD-related quality of life are worse in LPD subjects highlights the importance of this finding for counseling patients. Our results also add to the sparse literature regarding the durability of these effects showing persistent differences at 48 months in many of the same measures that differed at baseline. An additional interesting finding was that the LPD group had the diagnosis of PD for $\sim 1$ year longer than those without left-predominant disease. This observation may be related to their higher disease burden, which could ostensibly lead individuals to seek medical attention and thus arrive at a diagnosis earlier than their non-left dominant counterparts. The longer disease duration does not, however, explain the increased disease burden or faster progression of MDS-UPDRS part II scores because we controlled for disease duration in our analysis.

Our sensitivity analysis validated the direction of the main findings but revealed that this difference between NLPD and LPD progression and severity at 48 months held up only for the group with milder symptoms at baseline. While our study does not permit drawing firm conclusions about why this was so, we speculate that because more advanced cases (i.e., those with high baseline MDS-UPDRS II scores), have more extensive Lewy body pathology on both sides of the brain, the clinical differences between NLPD and LPD are therefore no longer present.

Our study had several limitations, most importantly that handedness was not documented in the original data set which prevented us from grouping subjects into dominant vs. nondominant sides of predominance. However, since $90 \%$ of the population is right-handed (30), this likely did not affect the results. Other limitations included our need to censor MDSUPDRS part III data from one site, variable lengths of follow-up of participants requiring us to model the subject-specific rates of progression using statistical techniques, and the lack of a universally agreed-upon method for determining asymmetry in PD. For instance, the method we used lacks sensitivity to detect asymmetry in very mild cases due to the ceiling effect, while alternative methods such as the UPDRS asymmetry index $(14,31)$ lack discriminant sensitivity at the high end of the MDS-UPDRS scale when subjects have more severe disease.

Though our study supports previous work showing a higher disease burden in LPD patients, and adds the finding of increased progression rate in MDS-UPDRS part II score, it remains unclear why LPD patients do worse. One potential biological explanation may be the existence of an increased dopaminergic reserve or more efficient motor network in the dominant left hemisphere of the 90 percent of individuals who are right-handed (30), thereby delaying the manifestations of motor symptoms on the contralateral side of the body (32). Another possible explanation is that more strenuous exercise and frequency of use of the dominant side of the body may be neuroprotective of dopamine networks thus accounting for the higher disease burden on the non-dominant side. This theory was bolstered by an experiment conducted in a rat model of PD created by unilateral injection of 6-hydroxydopamine into the striatum resulting in contralateral limb dysfunction and preferential use of the good limb. Investigators noted that when the healthy limb was immobilized forcing the rat to use the impaired limb, the expected contralateral limb dysfunction was prevented suggesting that frequency of use of the affected limb plays a significant role in motor dysfunction related to neurodegeneration (33).

The more rapid symptom progression and greater motor and non-motor symptom severity at baseline and 48 months are important clinical considerations. This information can be used for counseling patients regarding prognosis in a disease with notorious heterogeneity. Beyond this, the ability to determine which patients may progress at a more rapid rate in the future could prove invaluable in the elusive quest for disease-modifying therapy for PD where many therapeutics have been tested and failed. A major obstacle to demonstrating disease-modifying activity of a drug is the variable rate of symptom progression in study subjects which thus requires either a very large number of subjects or a long period of study or both. By recruiting study participants who are likely to have a rapid rate of progression, investigators may be able to more quickly identify the signal of disease-modifying activity. While lateralization of motor signs is not likely to be a sufficiently powerful predictor on its own for identification of fast progressors, it may wellserve as one of several selection criteria which could improve future clinical trial designs.

\section{CONCLUSION}

Our study provides evidence from a longitudinally-followed cohort of PD subjects that left-predominant motor symptoms are associated with more rapid progression of symptoms and a higher disease burden at baseline and 48 months. These findings provide valuable insight into the potential disease course of an individual patient and may prove helpful for patient counseling and for recruiting likely fast progressors into clinical trials of disease modifying agents. Further prospective studies in independent cohorts are needed to confirm and extend these observations.

\section{DATA AVAILABILITY STATEMENT}

Publicly available datasets were analyzed in this study. This data can be found here: https://pdbp.ninds.nih.gov/parkinsons-data.

\section{ETHICS STATEMENT}

The studies involving human participants were reviewed and approved by University of Texas Southwestern Medical Center 
IRB and by the IRBs at the other institutions who recruited subjects for this study. The patients/participants provided their written informed consent to participate in this study.

\section{AUTHOR CONTRIBUTIONS}

ME: study conception and draft manuscript preparation. ME and RD: study design, analysis and interpretation of results, and manuscript editing. JW: data analysis and statistical computation and statistical methods section writing. All authors reviewed the results and approved the final version of the manuscript.

\section{FUNDING}

This study was funded by NINDS U01-NS082148 and the Jean Walter Center for Research in Movement Disorders.

\section{ACKNOWLEDGMENTS}

Data and biospecimens used in preparation of this manuscript were obtained from the Parkinson's Disease Biomarkers Program

\section{REFERENCES}

1. Fahn S, Jankovic J, Hallett M. Chapter 4 - Parkinsonism: clinical features and differential diagnosis. In: Fahn S, Jankovic J, Hallett M, editors. Principles and Practice of Movement Disorders, 2nd Edn. Edinburgh: W.B. Saunders (2011). p. 66-92. doi: 10.1016/B978-1-4377-2369-4.00004-4

2. Sieber B-A, Landis S, Koroshetz W, Bateman R, Siderowf A, Galpern WR, et al. Prioritized research recommendations from the National Institute of Neurological disorders and stroke parkinson's disease 2014 conference. Ann Neurol. (2014) 76:469-72. doi: 10.1002/ana.24261

3. Fereshtehnejad S-M, Postuma RB. Subtypes of parkinson's disease: what do they tell us about disease progression? Curr Neurol Neurosci Rep. (2017) 17:34. doi: 10.1007/s11910-017-0738-x

4. Nutt JG. Motor subtype in Parkinson's disease: different disorders or different stages of disease? Mov Disord. (2016) 31:957-61. doi: 10.1002/mds. 26657

5. Kong XZ, Mathias SR, Guadalupe T, Glahn DC, Franke B, Crivello F, et al. Mapping cortical brain asymmetry in 17,141 healthy individuals worldwide via the ENIGMA Consortium. Proc Natl Acad Sci USA. (2018) 115:E5154-63. doi: 10.1073/pnas.1718418115

6. Convery R, Mead S, Rohrer JD. Review: clinical, genetic and neuroimaging features of frontotemporal dementia. Neuropathol Appl Neurobiol. (20190 45:6-18. doi: 10.1111/nan.12535

7. Wachinger C, Nho K, Saykin AJ, Reuter M, Rieckmann A. A longitudinal Imaging genetics study of neuroanatomical asymmetry in alzheimer's disease. Biol Psychiatry. (2018) 84:522-30. doi: 10.1016/j.biopsych.2018.04.017

8. Minkova L, Habich A, Peter J, Kaller CP, Eickhoff SB, Klöppel S. Gray matter asymmetries in aging and neurodegeneration: a review and meta-analysis. Hum Brain Mapp. (2017) 38:5890-904. doi: 10.1002/hbm.23772

9. Postuma RB, Berg D, Stern M, Poewe W, Olanow CW, Oertel W, et al. MDS clinical diagnostic criteria for Parkinson's disease. Mov Disord. (2015) 30:1591-601. doi: 10.1002/mds.26424

10. Zhong Z, Merkitch D, Karaman MM, Zhang J, Sui Y, Goldman JG, et al. High-spatial-resolution diffusion MRI in parkinson disease: lateral asymmetry of the substantia Nigra. Radiology. (2019) 291:149-57. doi: 10.1148/radiol.2019181042

11. Riederer P, Jellinger KA, Kolber P, Hipp G, Sian-Hülsmann J, Krüger R. Lateralisation in Parkinson disease. Cell Tissue Res. (2018) 373:297-312. doi: 10.1007/s00441-018-2832-z
(PDBP) Consortium, supported by the National Institute of Neurological Disorders and Stroke at the National Institutes of Health. Investigators include: Roger Albin, Roy Alcalay, Alberto Ascherio, Thomas Beach, Sarah Berman, Bradley Boeve, F. DuBois Bowman, Shu Chen, Alice Chen-Plotkin, William Dauer, Ted Dawson, Paula Desplats, Richard Dewey, Ray Dorsey, Jori Fleisher, Kirk Frey, Douglas Galasko, James Galvin, Dwight German, Lawrence Honig, Xuemei Huang, David Irwin, Kejal Kantarci, Anumantha Kanthasamy, Daniel Kaufer, James Leverenz, Carol Lippa, Irene Litvan, Oscar Lopez, Jian Ma, Lara Mangravite, Karen Marder, Laurie Orzelius, Vladislav Petyuk, Judith Potashkin, Liana Rosenthal, Rachel SaundersPullman, Clemens Scherzer, Michael Schwarzschild, Tanya Simuni, Andrew Singleton, David Standaert, Debby Tsuang, David Vaillancourt, David Walt, Andrew West, Cyrus Zabetian, Jing Zhang, and Wenquan Zou. The PDBP Investigators have not participated in reviewing the data analysis or content of the manuscript. We also thank Drs. Shilpa Chitnis, Pravin Khemani and Neepa Patel for helping to recruit and follow participants, and Ashley Gerald for her coordination of the study at UT Southwestern.

12. Amick MM, Grace J, Chou KL. Body side of motor symptom onset in Parkinson's disease is associated with memory performance. J Int Neuropsychol Soc. (2006) 12:736-40. doi: 10.1017/S1355617706060875

13. Dewey RB Jr, Taneja A, McClintock SM, Cullum CM, Dewey RB III, Bernstein I, et al. Motor symptoms at onset of Parkinson disease and risk for cognitive impairment and depression. Cogn Behav Neurol. (2012) 25:115-20. doi: 10.1097/WNN.0b013e31826dfd62

14. Cubo E, Martínez-Martín P, González-Bernal J, Casas E, Arnaiz S, Miranda J, et al. Effects of motor symptom laterality on clinical manifestations and quality of life in Parkinson's Disease. J Parkinsons Dis. (2020) 10:1611-20. doi: 10.3233/JPD-202067

15. Rosenthal LS, Drake D, Alcalay RN, Babcock D, Bowman FD, Chen-Plotkin A, et al. The NINDS Parkinson's disease biomarkers program. Mov Disord. (2016) 31:915-23. doi: 10.1002/mds.26438

16. Gottipati G, Karlsson MO, Plan EL. Modeling a composite score in Parkinson's Disease using item response theory. AAPS J. (2017) 19:837-45. doi: 10.1208/s12248-017-0058-8

17. Holden SK, Finseth T, Sillau SH, Berman BD. Progression of MDS-UPDRS scores over five years in de novo parkinson disease from the parkinson's progression markers initiative cohort. Mov Disord Clin Pract. (2018) 5:47-53. doi: $10.1002 / \mathrm{mdc} 3.12553$

18. Harrison MB, Wylie SA, Frysinger RC, Patrie JT, Huss DS, Currie LJ, et al. UPDRS activity of daily living score as a marker of Parkinson's disease progression. Mov Disord. (2009) 24:224-30. doi: 10.1002/mds.22335

19. Goetz CG, Tilley BC, Shaftman SR, Stebbins GT, Fahn S, MartinezMartin P, et al. Movement disorder society-sponsored revision of the Unified Parkinson's Disease Rating Scale (MDS-UPDRS): scale presentation and clinimetric testing results. Mov Disord. (2008) 23:2129-70. doi: $10.1002 / \mathrm{mds} .22340$

20. Nasreddine ZS, Phillips NA, Bédirian V, Charbonneau S, Whitehead V, Collin I, et al. The montreal cognitive assessment, MoCA: a brief screening tool for mild cognitive impairment. J Am Geriatr Soc. (2005) 53:695-9. doi: 10.1111/j.1532-5415.2005.53221.x

21. Tomlinson CL, Stowe R, Patel S, Rick C, Gray R, Clarke CE. Systematic review of levodopa dose equivalency reporting in Parkinson's disease. Mov Disord. (2010) 25:2649-53. doi: 10.1002/mds.23429

22. Ramaker C, Marinus J, Stiggelbout AM, Van Hilten BJ. Systematic evaluation of rating scales for impairment and disability in Parkinson's disease. Mov Disord. (2002) 17:867-76. doi: 10.1002/mds.10248 
23. Fereshtehnejad SM, Zeighami Y, Dagher A, Postuma RB. Clinical criteria for subtyping Parkinson's disease: biomarkers and longitudinal progression. Brain. (2017) 140:1959-76. doi: 10.1093/brain/awx118

24. Johns MW. A new method for measuring daytime sleepiness: the Epworth sleepiness scale. Sleep. (1991) 14:540-5. doi: 10.1093/sleep/ 14.6 .540

25. Doty RL, Shaman P, Kimmelman CP, Dann MS. University of Pennsylvania smell identification test: a rapid quantitative olfactory function test for the clinic. Laryngoscope. (1984) 94:176-8. doi: 10.1288/00005537-198402000-00004

26. Hamilton M. The assessment of anxiety states by rating. $\mathrm{Br} J$ Med Psychol. (1959) 32:50-5. doi: 10.1111/j.2044-8341.1959.tb00467.x

27. Hamilton M. A rating scale for depression. J Neurol Neurosurg Psychiatry. (1960) 23:56-62. doi: 10.1136/jnnp.23.1.56

28. Peto V, Jenkinson C, Fitzpatrick R. PDQ-39: a review of the development, validation and application of a Parkinson's disease quality of life questionnaire and its associated measures. J Neurol. (1998) 245:S10-4. doi: 10.1007/PL00007730

29. Poletti M, Frosini D, Pagni C, Baldacci F, Giuntini M, Mazzucchi $\mathrm{S}$, et al. The relationship between motor symptom lateralization and cognitive performance in newly diagnosed drug-naïve patients with Parkinson's disease. J Clin Exp Neuropsychol. (2013) 35:124-31. doi: 10.1080/13803395.2012.751966

30. Papadatou-Pastou M, Ntolka E, Schmitz J, Martin M, Munafò MR, Ocklenburg S, et al. Human handedness: a meta-analysis. Psychol Bull. (2020) 146:481-524. doi: 10.1037/bul0000229

31. Yogev G, Plotnik M, Peretz C, Giladi N, Hausdorff JM. Gait asymmetry in patients with Parkinson's disease and elderly fallers: when does the bilateral coordination of gait require attention? Exp Brain Res. (2007) 177:336-46. doi: 10.1007/s00221-006-0676-3
32. Ham JH, Lee JJ, Kim JS, Lee PH, Sohn YH. Is dominant-side onset associated with a better motor compensation in Parkinson's Disease? Mov Disord. (2015) 30:1921-5. doi: 10.1002/mds. 26418

33. Cohen AD, Tillerson JL, Smith AD, Schallert T, Zigmond MJ. Neuroprotective effects of prior limb use in 6-hydroxydopaminetreated rats: possible role of GDNF. J Neurochem. (2003) 85:299-305. doi: 10.1046/j.1471-4159.2003.01657.x

Conflict of Interest: RD reports personal fees (consulting) from Amneal, Acorda, Supernus, Teva, Adamas, US WorldMeds, Acadia, and Lundbeck, outside the submitted work.

The remaining authors declare that the research was conducted in the absence of any commercial or financial relationships that could be construed as a potential conflict of interest.

Publisher's Note: All claims expressed in this article are solely those of the authors and do not necessarily represent those of their affiliated organizations, or those of the publisher, the editors and the reviewers. Any product that may be evaluated in this article, or claim that may be made by its manufacturer, is not guaranteed or endorsed by the publisher.

Copyright (C) 2021Elkurd, Wang and Dewey. This is an open-access article distributed under the terms of the Creative Commons Attribution License (CC BY). The use, distribution or reproduction in other forums is permitted, provided the original author(s) and the copyright owner(s) are credited and that the original publication in this journal is cited, in accordance with accepted academic practice. No use, distribution or reproduction is permitted which does not comply with these terms. 